\section{Endoscopic Mucosectomy of the Esophagus with a "Shark-Tooth" Polypectomy Snare}

Simplified endoscopic resection of the esophageal mucosa using a cap-fitted panendoscope had been described by Inoue et al. (1). We report here on the use of a new type of polypectomy snare in the course of mucosectomy.

A 43-year-old woman presented with weight loss. Panendoscopy demonstrated a reddish flat area with a diameter of $7 \mathrm{~mm}$ in the esophagus, $28 \mathrm{~cm}$ from the incisors. The area was stained endoscopically with a $1 \%$ toluidine blue solution. Both the visible lesion and three other areas near it, each $5-6 \mathrm{~mm}$ in diameter and lying far apart, were stained. The largest one was removed completely by biopsy; the histology confirmed squamous-cell cancer. Endoscopic ultrasonography of the esophagus was negative. Since we considered that the lesions in the esophagus represented a multifocal intraepithelial cancer, we therefore decided to carry out an endoscopic mucosectomy in order to remove the lesions. At the endoscopic examination, the mucosal area was slightly hyperemie where the biopsy had been taken, but it did not stain with toluidine blue solution. The other three lesions became visible once again only after staining. Using an endoscopic injector, a physiological saline solution containing epinephrine 1:10,000 was injected underneath the lesions. The protruding polyp-like lesions were then grasped one by one with the "sharktooth" Olympus SBI6-LE polypectomy snare (Figure 1a), and removed by high-frequency diathermic cutting (Figure $1 \mathrm{~b}$ ). The histological examination of the lesions showed moderate dysplasia. and in one lesion also hyperkeratosis. A control panendoscopy and esophageal biopsy one month later were negative.

In our opinion. the use of toluidine blue solution prior to mucosectomy is advantageous, because the long-lasting staining of the lesions makes mucosectomy easier to perform. The use of the "shark-tooth" polypectomy snare made it easier to grasp the mucosal lesions and pull them into the esophageal lumen (Figure 2 ). The use of this snare is recommended when a transparent endoscope eap is not available, or if the diameter of the lesion is too large.

1. Szamto', J. Kiss', A. Vörös', A. Altorjay', P. Nagy

Dept. of Surgery

2Institute of Pathology, Postgraduate Medical School, Budapest,

Hungary

\section{Reference}

1. Inoue H, Takeshita K. Hori H, et al. Endoscopic mucosal resection with a cap-fitted panendoscope for esophagus. stomach, and colon mucosal lesion. Gastrointest Endose 1993; 39: $58-62$.

Corresponding Author

I. Szantó, M.D.

Dept, of Surgery

Postgraduate Medical School

Szaboles u 35. P.O. Box 112

1389 Budapest

Hungary

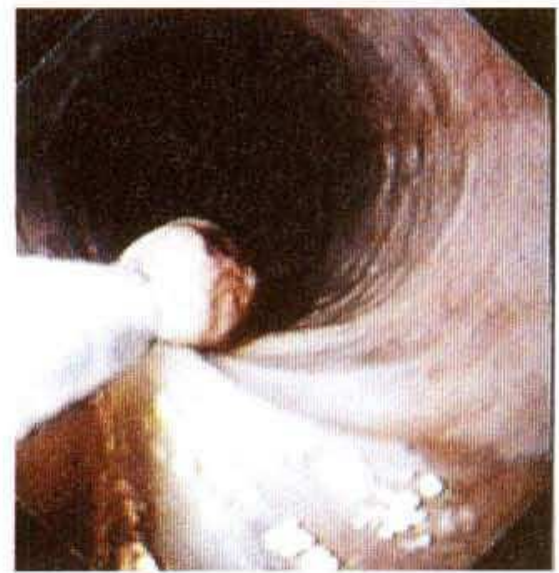

Figure 1: Mucosectorny technique. a Catching the lesion with the polypectomy snare.

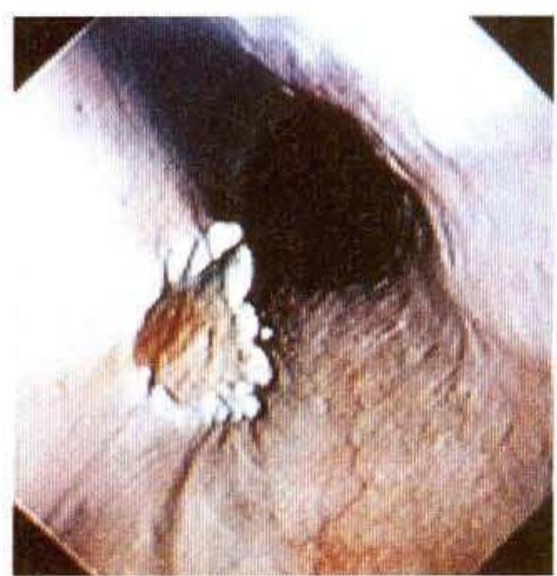

Figure $1 \mathrm{~b}$ site of the mucosectomy

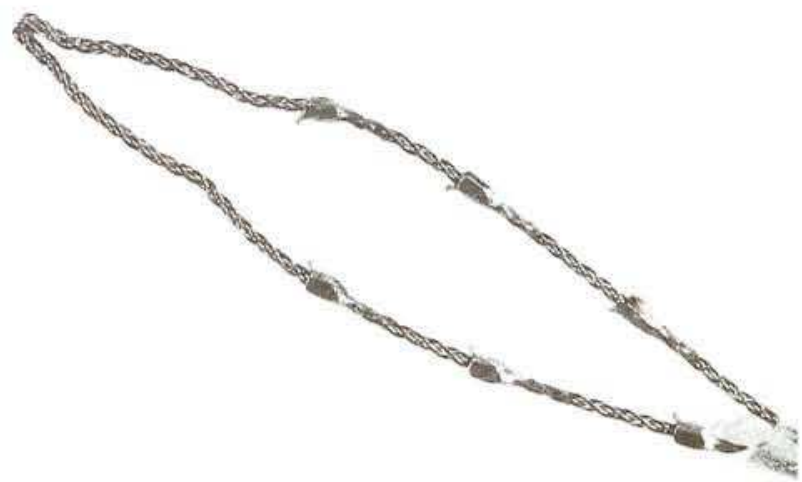

Figure 2: The "shark-tooth" polypectomy snare 